The compendium of self-enactable techniques to change and self-manage motivation and behaviour (v1.0)

5

6

7

8

Keegan Knittle, Matti Heino, Marta M. Marques, Minna Stenius, Marguerite Beattie, Franziska Ehbrecht, Martin S. Hagger, Wendy Hardeman, Nelli Hankonen

10

11

12

13

14

15

16

17

18 
20 Behaviour change techniques describe the content of behaviour change interventions, but do not adequately account for the actions that individuals must themselves undertake to successfully change or self-manage motivation or behaviour. This compendium of 123 self-enactable techniques fills this gap by combining behaviour- and motivation-regulation techniques across six existing classifications of behaviour change techniques and three scoping reviews, converting each into a self-enactable form with instructive examples to facilitate self-enactment. Qualitative feedback was gathered from intervention developers and the general public to improve the utility, congruence, and ease of self-enactability of technique definitions and instructive examples. This integrative index of self-enactable techniques can help intervention developers and individuals select appropriate selfdirected techniques to self-manage motivation and behaviour. Future research with this compendium will expand on the number of behaviours covered by the instructive examples and link techniques with their potential impacts on theoretical determinants of behaviours.

Supplementary Files (available from https://osf.io/pqfiz/):

\begin{tabular}{|l|l|}
\hline $\mathbf{1}$ & Simplified list version of the compendium (.pdf) \\
\hline $\mathbf{2}$ & Instructions and glossary for the general public (.pdf) \\
\hline $\mathbf{3}$ & Full tabular version of the compendium (.xlsx) \\
\hline $\mathbf{4}$ & Work scoping review (.pdf) \\
\hline $\mathbf{5}$ & Sport scoping review (.pdf) \\
\hline $\mathbf{6}$ & Education scoping review (.pdf) \\
\hline $\mathbf{7}$ & Preliminary compendium v0.3 - Tested in expert review (.xlsx) \\
\hline $\mathbf{8}$ & Verbatim methods of expert review exercise (.pdf) \\
\hline $\mathbf{9}$ & Full results of expert review (.xlsx) \\
\hline $\mathbf{1 0}$ & Notes of discussion after expert review (.pdf) \\
\hline $\mathbf{1 1}$ & Full methods and results of qualitative interviews (.pdf) \\
\hline
\end{tabular}


To be effective, many behaviour change interventions require that individuals take active roles in changing their own behaviour, by adopting and using appropriate strategies and methods (e.g., weighing pros and cons of changing, setting goals) to do so. This entails that individuals need to be equipped with the necessary skills, abilities, tools and techniques to effectively change their own behaviour, a process collectively referred to as self-management or self-regulation. While behaviour change interventions undoubtedly take place within complex systems involving many parts, there is evidence that individual-level interventions can change health-related (and other) behaviours ${ }^{1}$ and outcomes ${ }^{2}$, and these interventions are increasingly being used to reduce costs in healthcare systems across Western societies ${ }^{3}$. As such, the keys to improving health and well-being, as well as other issues which arise from the behaviour of individuals ${ }^{4}$, lie in the capabilities that individuals have at their disposal to successfully self-manage their own motivation and behaviour.

Behaviour change interventions often draw from behavioural theories, and target changes in important determinants of a behaviour to change the behaviour itself ${ }^{5}$. Historically, the descriptions of such interventions have lacked specificity, as broad treatment labels such as "cognitive therapy" or "lifestyle counselling" do not immediately reveal an intervention's component parts ${ }^{6}$. For example, two interventions with the same overarching label might contain different techniques, while, at the same time, two interventions with identical component techniques might receive different overarching treatment labels. This lack of granularity in intervention descriptions has led to a 'black box' problem in intervention research ${ }^{7}$, and has limited the scientific understanding of which 'active ingredients' effectively change behaviour within interventions.

Behaviour change techniques are the active components of behaviour change interventions ${ }^{8}$, and have been enumerated in several recently-developed taxonomies of behaviour change techniques. These include the 93-item behaviour change techniques taxonomy v1 (BCTTV1); the 99 -item intervention mapping (IM) taxonomy ${ }^{10}$, which arranges behaviour change techniques (or 'methods,' in IM terminology) by the theoretical determinants that each is presumed to target as a precursor to behaviour change; and the 38-item motivational interviewing (MI) taxonomy ${ }^{11}$, which specifies the content-based and relational techniques present within MI counselling approaches ${ }^{12}$. Taken together, these taxonomies ${ }^{9-11}$ offer researchers and practitioners an elaborated classification of the many methods available to change behaviours and some common language with which they can describe the content of behaviour change interventions. This has led to improved consistency in the description of behaviour change interventions, allowing for greater replicability of interventions, and offers those aiming to synthesize evidence across intervention studies means to adequately compare and classify intervention content. Across taxonomies, however, several shortcomings remain, including a lack of focus on individuals and technique enactment, limited scope, and insufficient examples of use.

\section{Shortcomings of existing taxonomies}

The most important outstanding issue is what the recipients of behaviour change interventions (i.e. individuals in the target population whose behaviour needs to change) can do on their own to facilitate behavioural change and maintenance. While some existing taxonomies indicate that techniques may be self-delivered ${ }^{9}$, the definitions and examples they provide focus largely on the actions that intervention providers (e.g., nurses, community workers, designers of public health campaigns) would take when delivering a technique to individuals (e.g., prompting behavioural goal setting, demonstrating the target behaviour, or providing information). This leaves 
how that should occur ${ }^{14}$. For example, within the BCTTv1, technique 11.2 (reduce negative emotions) states that intervention providers should "advise on ways of reducing negative emotions" to facilitate behavioural performance, but does not elucidate the actions that recipients of that technique would need to take in order to bring about change. In other words, how should an individual go about reducing their own negative emotions? Furthermore, some techniques from existing taxonomies do not lend themselves to self-enactment at all. This includes techniques from the IM taxonomy ${ }^{10}$ and TIPPME intervention typology ${ }^{15}$, which apply only to actors at other environmental levels (e.g., public policy or organizational change methods), and relational techniques from $\mathrm{Ml}^{12}$, which are only applicable to those delivering $\mathrm{Ml}$ in one-on-one practitionerclient sessions. While one existing taxonomy (the Oxford Food and Activity Behaviours taxonomy $\mathrm{OxFAB})^{16}$ has focused on self-enacted behaviour change and self-management, its techniques were drawn exclusively from weight management protocols, and have unknown applicability to other behavioural domains. The present study aims to compile a new domain-general list of techniques which focuses specifically on agentic and self-enactable behaviour change methods, which will offer intervention developers and the general public a clearer overview of the available options and specific actions which contribute to the successful self-management of behaviours related to health, environmental protection, and other outcomes.

Furthermore, by focusing primarily on behaviour change techniques that are delivered within interventions, existing taxonomies also do not specifically address the issue of technique enactment, which is imperative when investigating the behaviour change of individuals within complex systems ${ }^{17,18}$. For an intervention to have effects, providers must successfully deliver techniques, individuals must successfully receive the techniques, and individuals must then also successfully act on the techniques ${ }^{19}$. A growing body of evidence suggests that sustained behaviour change following interventions depends on the extent to which individuals self-enact or utilise behaviour change techniques themselves ${ }^{20-22}$, but existing taxonomies do not indicate what successful self-enactment should look like, or which techniques require enactment beyond delivery. This may limit the extent to which intervention developers can take the individual perspective into account when trying to optimize enactment. This work will therefore also promote intervention designs which more coherently address enactment, and which bridge the gap between intervention receipt and the adoption and maintenance of new behaviours.

Second, existing technique classifications do not capture all possible techniques that might be used to change or regulate behaviour or behavioural determinants (e.g., motivation), so drawing techniques from a wider range of behavioural domains could reveal additional techniques. For example, within work and occupational psychology, the idea that individuals can themselves alter working patterns to increase their own motivation ('job craft') has attracted much attention. The job crafting research to date indicates that various positive outcomes follow when employees themselves make changes to their work patterns and relationships ${ }^{23}$. Within sport psychology a number of studies have linked cognitive self-management strategies, such as self-talk, imagery, and attentional focus with positive outcomes ${ }^{24,25}$. Attentional focus is also prevalent as a selfmanagement technique within educational psychology. This work will therefore explore selfmanagement and behaviour change strategies in various applied domains, which could unearth new techniques to supplement existing taxonomies and make them more complete.

Finally, while existing taxonomies offer some examples of how techniques might be applied in practice, these are generally limited in scope and described using technical terminology. This makes the meaning and operationalisation of individual techniques less accessible and 
comprehensible to practitioners who may lack expertise in behavioural science. It also means that, while techniques from existing taxonomies might potentially be self-enactable, self-enactment would not likely occur successfully in practice. The current work aims to increase the likelihood of successful self-enactment, by writing self-enactable techniques in plain, accessible language and by including adequate instructions and examples to facilitate ease of use by the general public.

\section{The Present Study}

The present study aimed to develop an integrative compendium of self-enactable techniques to change or self-manage motivation and behaviour, with a focus on techniques which require conscious participation and initiation on the part of the individual. Specifically, this research will (a) identify, assess, and integrate techniques across existing taxonomies and other domains of psychological research (sport, education and work); (b) identify how individuals can take an active role in enacting the identified techniques to change or manage motivation and behaviour; and (c) compile a comprehensive list of self-enactable behaviour change techniques that intervention developers can adopt to incorporate into interventions aimed at changing motivation and behaviour. To achieve this, our group undertook an iterative development process that involved searching and content-analysing existing research on behaviour change interventions and extant taxonomies; discussions within the research team and an advisory group comprising behaviour change experts; feedback from experienced intervention developers; and qualitative interviews with members of the public. This process involved: identifying relevant techniques; outlining how the techniques could be self-enacted and developing appropriate definitions, descriptions, and self-enactable formats; producing instructive examples; and identifying information about dependencies between techniques. Table 1 outlines the steps taken during this research, and further details are available in the methods section.

\section{Results \\ Developing the compendium of self-enactable behaviour changes involved three distinct phases: Initial development work (Phase 1); external expert reviews (Phase 2); and the refinement of the compendium into its final form (Phase 3 ). The Methods section provides further detail on the processes undertaken during the three phases (and seven individual steps) shown in Table 1.}

\section{Phase 1: Initial development}

In step one, the 230 techniques from the three primary source taxonomies ${ }^{9-11}$ were consolidated in a spreadsheet, leading to a provisional listing (v0.1) containing 125 techniques. This provisional listing was then supplemented with the 13 additional techniques shown in Table 2, which were derived from three scoping reviews (in the areas of work, sport and education psychology (Step 2; Supplementary Files 4, 5 and 6), and three additional classifications of behaviour change techniques ${ }^{16,26,27}$ (Step 3). Steps two and three resulted in an expanded provisional listing of 138 techniques (v0.2). Finally, in step four, the text of each technique in v0.2 was re-written into a selfenactable form and supplemented with a plain-language instructive example of how to self-enact it. This resulted in a first draft of the compendium (v0.3) which contained 123 techniques. Figure 1 shows the flow of techniques from original sources through to the final compendium, and specifies reasons for removal of techniques. 
In step five, we conducted qualitative interviews with members of the general public (i.e., one group of potential end users of the compendium) to examine the acceptability of a subset of the techniques from version 0.3 . These interviews revealed several issues with the definitions and examples of some techniques, which limited their potential acceptability as part of self-enacted behaviour change interventions. The interviews identified the presence of technical language which interviewees had difficulty understanding. Some interviewees expressed doubts about the personal relevance of some techniques (e.g., "I could see how this might be good for someone else, but not me"). Some interviewees found it difficult to identify ways to implement the techniques beyond what was explicitly mentioned in the technique definitions or examples.

Table 1. Outline of the steps taken in developing the compendium of self-enactable techniques.

\begin{tabular}{|c|c|c|c|}
\hline Phase & Step & Methods & Outputs \\
\hline \multirow[t]{4}{*}{$\begin{array}{l}\text { 1. Initial } \\
\text { development }\end{array}$} & $\begin{array}{l}\text { 1. Integrating three existing } \\
\text { global taxonomies of } \\
\text { behaviour change } \\
\text { techniques/methods }\end{array}$ & $\begin{array}{l}\text { Group discussions within } \\
\text { research team; consultations } \\
\text { with authors of previous } \\
\text { technique classifications }\end{array}$ & $\begin{array}{l}\text { A provisional list of } \\
\text { technique definitions } \\
\text { (v0.1; } n=125 \text { ) }\end{array}$ \\
\hline & $\begin{array}{l}\text { 2. Identifying techniques } \\
\text { from applied psychology } \\
\text { literature }\end{array}$ & $\begin{array}{l}\text { Three scoping reviews of self- } \\
\text { management in the sport, } \\
\text { education and work psychology } \\
\text { domains }\end{array}$ & $\begin{array}{l}\text { Additional self- } \\
\text { enactable techniques } \\
\text { for potential inclusion. }\end{array}$ \\
\hline & $\begin{array}{l}\text { 3. Adding in content from } \\
\text { scoping reviews and other } \\
\text { previous (domain-specific) } \\
\text { classifications of behaviour } \\
\text { change techniques }\end{array}$ & $\begin{array}{l}\text { Group discussions within } \\
\text { research team; consultations } \\
\text { with authors of previous } \\
\text { technique classifications }\end{array}$ & $\begin{array}{l}\text { An expanded } \\
\text { provisional list of } \\
\text { technique definitions } \\
\text { (v0.2; } n=138 \text { ) }\end{array}$ \\
\hline & $\begin{array}{l}\text { 4. Creating instructive } \\
\text { examples to improve ease of } \\
\text { self-enactability }\end{array}$ & $\begin{array}{l}\text { Group discussions within } \\
\text { research team; consultations } \\
\text { with authors of previous } \\
\text { technique classifications and } \\
\text { other behaviour change experts }\end{array}$ & $\begin{array}{l}\text { A draft list of technique } \\
\text { definitions and } \\
\text { examples (v0.3; } n=123 \text { ) }\end{array}$ \\
\hline \multirow[t]{2}{*}{$\begin{array}{l}\text { 2. External } \\
\text { reviews }\end{array}$} & $\begin{array}{l}\text { 5. Assessing acceptability of } \\
\text { a subset of techniques }\end{array}$ & $\begin{array}{l}\text { Qualitative interviews with } \\
\text { members of the public }(n=20)\end{array}$ & $\begin{array}{l}\text { Possible improvements } \\
\text { of the definitions and } \\
\text { examples in v0.3 }\end{array}$ \\
\hline & $\begin{array}{l}\text { 6. Assessing utility, } \\
\text { congruence and ease of self- } \\
\text { enactability of technique } \\
\text { definitions and examples }\end{array}$ & $\begin{array}{l}\text { Online survey of external } \\
\text { experts in intervention } \\
\text { development }(n=17)\end{array}$ & $\begin{array}{l}\text { Possible improvements } \\
\text { of the definitions and } \\
\text { examples in v0.3 }\end{array}$ \\
\hline $\begin{array}{l}\text { 3. Refinement } \\
\text { and finalising }\end{array}$ & $\begin{array}{l}\text { 7. Improving technique } \\
\text { definitions, examples, and } \\
\text { overall usability }\end{array}$ & $\begin{array}{l}\text { Group discussions within } \\
\text { research team to reach } \\
\text { consensus on final wording of } \\
\text { technique definitions and } \\
\text { examples }\end{array}$ & $\begin{array}{l}\text { The final compendium } \\
\text { ( } v 1.0 ; n=123), \text { which } \\
\text { includes introductory } \\
\text { text and a glossary }\end{array}$ \\
\hline
\end{tabular}


Figure 1. Sources of identified techniques and the flow of techniques into the final compendium.

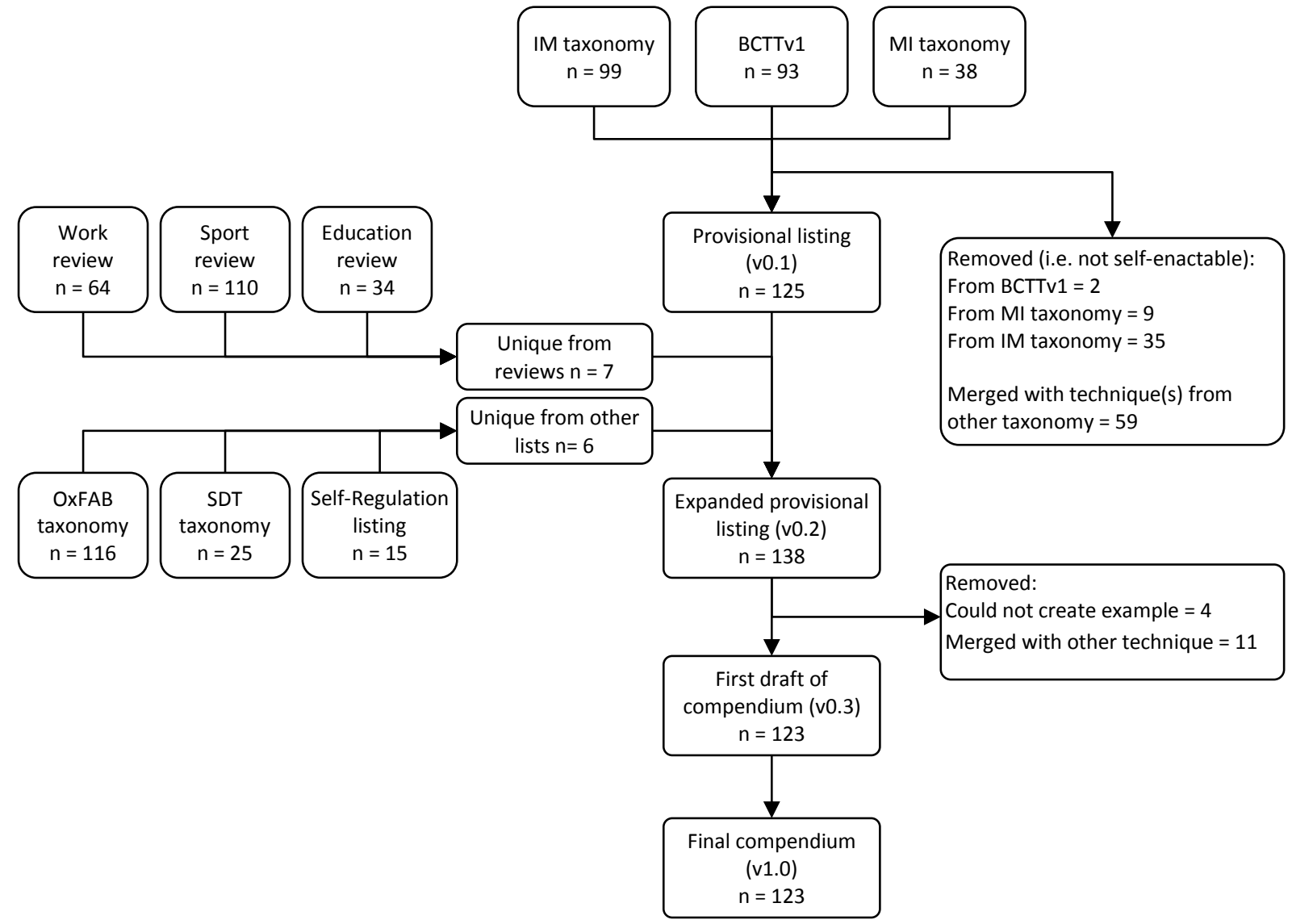

In step six, external experts in intervention development $(n=20)$, another group of potential end users of the compendium, used an online system to rate the labels, definitions, and examples of included techniques on three dimensions: utility, congruence, and ease of self-enactability. Experts also provided comments about how each technique, and the draft compendium as a whole, could be improved. Rates of agreement across experts ranged from $70.5 \%$ for utility, to $64.9 \%$ for congruence, to $53.7 \%$ for ease of self-enactability. We did not calculate Fleiss' kappa for multiple raters, as the review exercise aimed to identify possible problems with the techniques as written and did not aim to achieve a consensus or final agreed-on rating for each technique ${ }^{28}$. A breakdown of quantitative responses from the review exercise is presented in Figure 2 . In total, results of the expert review indicated that the utility of 28 techniques, the congruence of 34 techniques, and the ease of self-enactability of 62 techniques required improvements to the definitions and examples. Fifty-five techniques did not require improvement in any of these three dimensions, 28 needed improvement in one dimension, 24 needed improvement in two dimensions, and 16 needed improvement in all three dimensions. The results of Phase 2 indicated several concrete ways to improve the definitions and examples in Phase 3. 
Figure 2. Percentages of expert responses $(n=492)$ to questions of utility, congruence and ease of self-enactability for the definitions and examples in the draft compendium (v0.3). Definitions and examples of problematic techniques were then revised, resulting in the final compendium (v1.0).

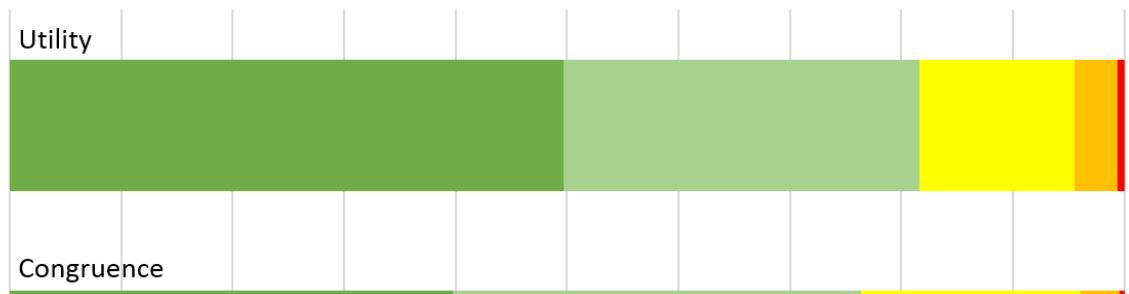

To what extent does the example reflect the definition?

To what extent would the average person be able to successfully use this technique after reading the definition and example?

\section{Phase 3: Refinements and finalizing}

The results of Phase 2 informed the final Phase of development, wherein we used qualitative feedback from intervention development experts and the general public to adapt the compendium into its final revised form. This involved rewriting definitions and instructive examples of problematic techniques to improve utility, congruence and ease of self-enactability. In addition, based on suggestions from the expert review, each technique was supplemented with information about possible unintended adverse effects, and information to distinguish between techniques that would likely require instruction on delivery, and those that are more readily and independently selfenactable based on the provided definitions and instructive examples alone. Finally, based on suggestions in the qualitative data, a primer and glossary were added to summarize the purposes of the compendium, to offer guidance on how to use it, and to define key terms from the technique definitions and examples. This resulted in the final compendium (v1.0; supplementary file 3).

\section{Discussion}

Taxonomies of behaviour change techniques provide a common set of terms for describing the unique components of behaviour change interventions, and improve the uniformity of descriptions to facilitate replicability and evidence synthesis. This integrative compendium of selfenactable techniques builds on existing taxonomies in three key ways: by reconceptualising techniques as actions that individuals can themselves undertake to change or self-manage motivation or behaviour; by combining techniques across existing taxonomies and from applied psychological research in the areas of work, sport, and education; and by including instructive examples, information about adverse effects and dependencies between techniques, and a guide to facilitate self-directed use of the techniques. These advances offer researchers and intervention developers a comprehensive resource for optimally accounting for the participant perspective when building behaviour change interventions, and have the potential to facilitate self-enactment of these 
techniques among the general public. This compendium is a first step in this direction (v1.0), and it will be further developed, expanded and updated as additional evidence comes to light.

The agentic approach taken within this work draws attention to the importance of fidelity of receipt and enactment within behaviour change interventions ${ }^{19}$. Many existing interventions are not delivered or enacted as intended, which can reduce the effectiveness of these interventions. By conceptualising techniques not only in terms of what is delivered, but also in terms of the concrete actions that individuals receiving an intervention must themselves take to bring about change, this work will help intervention developers to carefully consider and plan ways to increase fidelity of receipt and enactment. Further extensions to this compendium may include questionnaire items to assess enactment of techniques in a way that accounts for these actions ${ }^{16}$.

Behaviour change researchers may also find this compendium useful for coding the content of self-delivered interventions (e.g., self-help). Despite the known under-reporting problems associated with retrospective taxonomy-based coding of intervention content from published intervention descriptions ${ }^{29}$, this method is consistently used in meta-analyses across behavioural domains. While taking a self-enactment approach when coding published intervention descriptions will not solve the problem of under-reporting, it could help to identify subtle variations in the delivery of certain techniques, which may contribute to a technique's effectiveness across studies. Furthermore, if applied to the coding of individual interactions between intervention providers and recipients, an agentic approach could help to identify differences in technique enactment across participants within studies, by examining the extent to which providers bring up and discuss specific agentic actions participants took outside of sessions ${ }^{30}$.

The detailed instructive examples which accompany the techniques in this compendium will help to facilitate self-enactment. Each instructive example offers a rationale for using the technique, and lists concrete actions an individual should take to enact the technique to self-manage or change behaviour or motivation. While not a guarantee of successful self-enactment, these brief and informative instructive examples capture the essence of each technique, and have been reviewed and refined based on the inputs of intervention developers and members of the general public alike. This means that they meet a minimal threshold of prospective acceptability ${ }^{31}$, and could be used as off the shelf options in face-to-face or technology-assisted self-management or behaviour change interventions. However, this work does not yet provide evidence for the concurrent acceptability or efficacy of any techniques, and future testing is needed to examine how well individuals can selfenact these techniques based on these definitions and examples alone. With further refinements based on the results of such testing, the techniques could open new possibilities for self-delivered interventions. This is an important contribution, as effective self-delivered or technology-assisted interventions have great potential to reduce the costs associated with primary prevention and medical management of chronic disease ${ }^{32}$, and in improving other outcomes.

In addition to their utility for researchers and intervention developers, the instructive examples offer members of the public direct access to self-enactable techniques that they could use to self-manage or change their behaviour. This includes techniques that are best used before (e.g., obtaining information, mental rehearsal), during (e.g., action control, distraction), or after (e.g., reviewing behavioural goals, self-reward) engaging in a target behaviour. It also includes techniques that would be expected to change behaviour or motivation via reflective and deliberative processes (e.g., goal setting, graded tasks), and those that target automatic or impulsive response (e.g., habit formation, training executive function). As this work focused solely on agentic actions, we excluded 
awareness (e.g., choice architecture or nudging), as well as techniques which could not be reasonably self-initiated (e.g., policy-level interventions). We did, however, include techniques which might (potentially) require external inputs (e.g., from other people, the internet or healthcare professionals), but which individuals could nevertheless self-deploy (e.g., obtain social support); and techniques by which an individual might automatize their behavioural patterns (e.g. habit formation). The final listing distinguishes between techniques that might require external inputs and those which do not, and provides additional information about prerequisite techniques, to avoid self-enactment of techniques for which the necessary preconditions have not been met. The information accompanying each technique will be expanded in the future to include further information about each technique's parameters of effectiveness.

Based on our expert review and interviews with potential end users, we also added an introductory text to the compendium, which outlines how it can be used and defines several key terms from the behaviour change literature. While this accessibility and user-friendliness goes beyond that offered by existing taxonomies, which provide no such guidance to members of the public looking change their health behaviours on their own, it stops short of being a fully self-guided intervention platform. Rather, in its present form, the listing offers the general public a list of ideas for how to go about changing or managing their own lifestyle behaviours or motivation.

As the compendium at this point lacks the capability to fully guide individuals through the process of behaviour change, several areas of concern for misuse and unintended consequences of techniques require highlighting. During the expert review phase, several techniques were flagged as potentially having adverse effects when used incorrectly ${ }^{33}$, or when applied to a different behaviour than the ones included in the instructive examples. As an example, when the technique satiation (\#69) is targeted toward physical activity (i.e., sitting for an extended period until physical activity feels like a nice change from sitting), no immediate adverse events would be expected. However, when applied to reducing unhealthy snack intake, the technique could lead to unhealthy binge eating behaviours and potentially contribute to the development of eating disorders ${ }^{34}$. Although most potentially adverse effects from technique misuse were mild (e.g., frustration at not achieving a goal, placing a burden on friends), we found it important to proactively identify and clearly indicate these to individuals wishing to self-manage their behaviour, and have added such designations where applicable. While this is currently sufficient, future development of this compendium into a standalone system for self-delivered behaviour change interventions would necessitate a more complete plotting of worst-case scenarios and implementation of more rigorous safeguards to protect individuals who might unknowingly misapply these self-enactable techniques.

In developing this compendium, our research team followed a systematic and stepwise process that was informed by past experiences with taxonomy development ${ }^{11,35,36}$. This included extensive in-depth discussions and consensus-reaching procedures, scoping reviews, input from a panel of expert intervention developers, and input from authors of published taxonomies and other topic-area experts. The development process also included the novel aspect of qualitative interviews with the public to assess and improve the comprehensibility of a subset of techniques.

Despite these strengths, several limitations of this work related to both the final product and the development process bear mentioning. First, the instructive examples currently relate to only one or two health-related behaviours (e.g. physical activity, healthy eating, smoking cessation) per technique. During the expert review phase, several experts called for an extension of the examples to cover a wider range of health and environmental protection behaviours. This is important, as some techniques are better suited to changing some behaviours than they are others (e.g. 
differences between "stop" and "start" behaviours, behaviours with addictive elements). Given the wide range of behaviours that interventions might target, it was not feasible to extend the compendium beyond its current form within the current project. To expand this work in the future, our group has set up a crowdsourcing platform ${ }^{37}$, through which researchers and others can contribute their own examples of how each technique could be used to target health behaviours not currently covered. While facilitating this crowdsourcing approach presents quality control and logistical challenges, which themselves require resources to overcome, expanding on this work via a collaborative effort of the scientific community is an exciting possibility. We welcome submissions for new examples via the online form at http://bit.do/SubmitAnExample.

Second, while most of these techniques have been included as part of previous behaviour change interventions, this was rarely done in a specifically self-enactable form. There is therefore little evidence about the efficacy of these techniques when self-enacted. Instead of making claims about technique efficacy, this compendium of self-enactable techniques supports the development of self-enactable intervention components, the efficacy of which would need to be tested separately. Relatedly, this listing also does not include comprehensive information about how each technique relates to motivational constructs and other behavioural determinants. Other research groups are currently working to establish an evidence-base for the linkages between behaviour change techniques and behavioural determinants (i.e. an ontology of behaviour change) ${ }^{38,39}$, which may be tied into this work in the future.

Finally, interviews with members of the public about the perceived acceptability and utility of techniques only covered a subset of the techniques included here. While these interviews led to several improvements in these 20 techniques, we were unable to conduct interviews for all included techniques. Work is underway to expand upon the qualitative findings presented here, and any resultant improvements to technique definitions or instructive examples will be integrated into the compendium in due course (https://osf.io/pqfiz/). We would therefore like to echo previous calls for further research into uptake and enactment of behaviour change techniques ${ }^{40}$.

In taking this work further, one could envision an online system to offer members of the public guided, individualised access to these techniques. By utilising principles of computer tailoring ${ }^{41,42}$ and ongoing ontological work to improve the evidential links between behaviour change techniques and behavioural determinants ${ }^{38,39}$, such a system could account for individuals' current states and offer choices of the best techniques they could self-enact to change or manage their behaviour in real time. Paucity of research on some behaviour change techniques, especially when used in a self-enactable way, means that fully realising this type of evidence-based system would require substantial advances in the breadth and depth of the evidence base. However, such a system could also work to expand the evidence base on its own.

This compendium could also be used to develop measures of self-enactment processes for assessing fidelity within interventions. Measuring enactment of behaviour change techniques requires short technique definitions that can be readily utilised as questionnaire items. HartmannBoyce and colleagues have previously created a questionnaire based on their OxFAB taxonomy work ${ }^{16}$, and a similar process could be undertaken utilising the self-enactable techniques presented here. Developing adequate measures is key to improving scientific understanding of what individuals themselves do to change and manage their behaviour.

In conclusion, this integrative compendium of self-enactable techniques to change and selfmanage motivation and behaviour builds upon existing taxonomies of behaviour change techniques, 
and clarifies the agentic actions needed for successful self-enactment. It also extends previous taxonomies by pulling together their component techniques into a single listing, and by including clear instructions for how to use each technique in practice. In its present form, researchers can use this list to develop behaviour change interventions that optimally account for enactment by intervention recipients. This also offers members of the public access to definitions and instructive examples of self-enactable techniques that they could themselves use to change or manage their behaviour. With further refinements and contributions from theory and evidence, these intervention delivery and self-enactment perspectives could be brought together into a generalised, self-guided behaviour change system tailored to the needs of individuals.

\section{Methods}

\section{Step 1: Integrating existing primary taxonomies}

In creating this compendium of self-enactable techniques for motivation and behaviour change, the intervention mapping taxonomy $\mathrm{y}^{10}$, the BCTTV $1^{9}$, and the motivational interviewing taxonomy ${ }^{11}$ were chosen as primary sources, as they each identify and describe behaviour change techniques that are applicable across multiple behavioural domains. Efforts were then made to map these taxonomies onto one another (i.e., to combine them while accounting for overlaps). The mapping exercise was initiated by a single researcher (MB), who first placed the 93 techniques from the BCTTV1 into a spreadsheet. The BCTTV1 was used as the starting point, as it is extensively used within behaviour change intervention research. Then, each subsequent technique from the other two taxonomies was examined individually in relation to the techniques present in the BCTTV1. If a subsequent technique was judged to overlap (or partially overlap) with a technique present in the spreadsheet, then these techniques were mapped on to one another by placing the label of this new technique in the cell adjacent to the one containing the existing technique. If no match or overlap with the existing list was perceived, then a new row containing this new technique was added to the spreadsheet. In case of any uncertainty regarding the overlap of techniques from new sources, notes were made for later discussion with other members of the research team. The result of this mapping exercise and any uncertainties encountered were fully reviewed and discussed in detail until consensus on the mapping was reached within the study group ( $\mathrm{MB}, \mathrm{NH}, \mathrm{MH}, \mathrm{KK}, \mathrm{MS})$. Where consensus was not reached during discussions within this group, the study advisory group ( $M S H, W H, M M M)$, the authors of source taxonomies and additional topic experts were consulted via email, skype or in person for clarity on how they would differentiate between techniques from different taxonomies. These opinions informed further discussions within the study group to reach consensus.

After this initial mapping exercise, all techniques from the combined post-mapping list were evaluated for potential conversion into a self-enactable technique by a study group member (MH or KK). Techniques which were adjudged to have limited possibility of self-enactability were maintained and discussed with the rest of the research team. After these discussions, techniques were only removed due to lack of selfenactability when all members of the study group agreed the technique was not self-enactable.

In the next step, $\mathrm{MB}, \mathrm{NH}, \mathrm{MH}, \mathrm{KK}$ and $\mathrm{MS}$ (with inputs from MMM and WH) worked collaboratively to rewrite each technique definition in a self-enactable way, using three pre-specified criteria: First, each technique had to contain at least one verb (e.g., seek out, obtain, arrange, reflect on) that refers to the action an individual would need to take to self-enact the technique. Second, each rewritten technique had to refer to either the performance of, and/or motivation for, a specific target behaviour. This could include engaging in a wanted behaviour and/or refraining from engaging in an unwanted behaviour. Definitions were worded to accommodate both possibilities where applicable. Finally, all techniques were written under the assumption that an individual has already identified a specific target behaviour that they are considering changing or already desire to change. One technique (\#1 - Agenda mapping) was an exception to this rule however, as it involved choosing a behavioural domain. In writing the definitions, wordings present in the BCTTv1 were used 
as a guide, and these were supplemented or altered where necessary to accommodate self-enactment and to include operationalisations of techniques from other sources.

\section{Step 2: Scoping reviews to identify additional techniques}

Three scoping reviews were undertaken by MB and MS to identify potential additional techniques from the domains of sport, education, and work psychology. These scoping reviews included examining topicrelated reviews, interventions, theories and questionnaire items from each of these three domains. The full methods and findings of the scoping reviews in the work, sport, and education domains are reported in supplementary files 4,5 and 6 respectively, which are available on the project's open science framework page (https://osf.io/pgfiz/).

Table 2. Final forms of techniques added during Phase 1, Step 3 of the development process.

\begin{tabular}{|c|c|c|c|}
\hline Number & Label & Definition & Source \\
\hline 29 & Task crafting (enjoyment) & $\begin{array}{l}\text { Restructure the target behaviour to make } \\
\text { performing it more enjoyable }\end{array}$ & $\begin{array}{l}\text { OxFAB taxonomy; } \\
\text { Work scoping review }\end{array}$ \\
\hline 30 & $\begin{array}{l}\text { Task crafting (skills and } \\
\text { ability) }\end{array}$ & $\begin{array}{l}\text { Introduce new approaches to the target } \\
\text { behaviour that are congruent with current skills } \\
\text { and ability }\end{array}$ & Work scoping review \\
\hline 31 & Add challenge & Add challenges to the target behaviour. & Work scoping review \\
\hline 32 & Goal integration & $\begin{array}{l}\text { Modify (or choose ways of doing) the behaviour } \\
\text { such that it allows for simultaneously engaging } \\
\text { in other valued behaviours and/or pursuing } \\
\text { valued outcomes }\end{array}$ & $\begin{array}{l}\text { Work scoping review; } \\
\text { Group discussion }\end{array}$ \\
\hline 52 & Support others & $\begin{array}{l}\text { Provide support to others in relation to the } \\
\text { target behaviour }\end{array}$ & $\begin{array}{l}\text { OxFAB taxonomy; } \\
\text { Work scoping review }\end{array}$ \\
\hline 57 & $\begin{array}{l}\text { Remind of outcome goal } \\
\text { content }\end{array}$ & Remind yourself of your outcome goal(s). & $\begin{array}{l}\text { Work scoping review; } \\
\text { Group discussion }\end{array}$ \\
\hline 58 & $\begin{array}{l}\text { Action control (keep goals } \\
\text { in mind) }\end{array}$ & $\begin{array}{l}\text { Make efforts to consciously keep the target } \\
\text { behaviour and your goals in mind }\end{array}$ & $\begin{array}{l}\text { Self-regulation listing; } \\
\text { Sport scoping review }\end{array}$ \\
\hline 59 & $\begin{array}{l}\text { Action control (maximize } \\
\text { effort) }\end{array}$ & $\begin{array}{l}\text { Maximise effort toward undertaking the target } \\
\text { behaviour }\end{array}$ & $\begin{array}{l}\text { Self-regulation listing; } \\
\text { Sport scoping review; } \\
\text { Education scoping review }\end{array}$ \\
\hline 103 & Critically assess beliefs & $\begin{array}{l}\text { Evaluate and challenge the accuracy of your } \\
\text { own beliefs }\end{array}$ & Work scoping review \\
\hline 109 & $\begin{array}{l}\text { Focus on enjoyment } \\
\text { (pleasant aspects) of } \\
\text { behaviour }\end{array}$ & $\begin{array}{l}\text { Focus thinking on pleasant rather than } \\
\text { unpleasant aspects of the target behaviour. }\end{array}$ & Work scoping review \\
\hline 120 & $\begin{array}{l}\text { Identify sources of } \\
\text { pressure for behaviour }\end{array}$ & $\begin{array}{l}\text { Identify sources of pressure (external or } \\
\text { internal) and expectations to perform the target } \\
\text { behaviour }\end{array}$ & SDT taxonomy \\
\hline 121 & $\begin{array}{l}\text { Identify ways of dealing } \\
\text { with pressure }\end{array}$ & $\begin{array}{l}\text { Take steps to manage or limit the effects of } \\
\text { pressure (external or internal) to perform the } \\
\text { target behaviour }\end{array}$ & SDT taxonomy \\
\hline 123 & Prayer & $\begin{array}{l}\text { Appeal to a higher power for changes in } \\
\text { motivation or behaviour }\end{array}$ & Education scoping review \\
\hline
\end{tabular}



made notes on their possible overlaps with those already present in the merged taxonomy. These notes were then reviewed by additional members of the study team ( $\mathrm{NH}, \mathrm{MH}, \mathrm{MMM}$ ), and non-overlapping techniques were added to the existing list. Similarly, each technique from three additional classifications of behaviour change techniques ${ }^{16,26,36}$ was reviewed by at least one researcher (MB, NH, MH, KK, MMM). Techniques identified as potentially unique were then discussed by $\mathrm{NH}, \mathrm{MH}, \mathrm{KK}$, and $\mathrm{MMM}$ until consensus was reached on uniqueness or overlap with existing techniques in the listing. Authors of two secondary sources ${ }^{16,26}$ were contacted for additional information where consensus could not immediately be reached within the study group. Techniques added to the listing during Step 3 were reworded into a self-enactable form following the same procedures as in Step 1, after consensus had been reached on their inclusion (See Table 2).

\section{Step 4: Creating instructive examples}

Each technique from the expanded provisional listing (v0.2) was then supplemented by an instructive example which could allow the average person to self-enact the technique to change or self-manage a behaviour. While the techniques could, strictly speaking, be used to self-manage any behaviour, we elected to focus the contents of initial examples on health-related behaviours. To create the examples, five techniques from v0.2 were selected at random, and members of the study group ( $\mathrm{FE}, \mathrm{NH}, \mathrm{MH}, \mathrm{KK}, \mathrm{MMM}$ ) worked independently to create instructive examples for each of these same five techniques. The group then met to collaboratively discuss the positive and negative aspects of each of these independently-created instructive examples, and co-wrote instructive examples that best represented the five techniques in question. The characteristics of the resulting instructive examples, as well as the positives and negatives of the independently-created instructive examples were then worked into guidelines for the creation of subsequent instructive examples. The guidelines stated that each instructive example should: (1) be consistent with the technique's definition; (2) be written in an instructive way that would enable a lay person reading it to implement the technique on their own; and (3) refer to a specific health behaviour (e.g., physical activity, diet, smoking). Additionally, examples were required to follow a uniform structure: An introduction sentence; 2-3 concrete examples written in complete sentences with one sentence per example the standard; and an optional additional sentence with information on the best ways of doing the technique and/or its relation to other techniques. Furthermore, the created examples should not contain instructions that could constitute another technique, include any unnecessary verbs that are not put into action in the example (e.g., "Think about doing..." should simply be "do..."), or contain unnecessary linking words that might have unintended meanings (e.g., alternatively, conversely).

In the next step, a draft example was created for each technique by a randomly selected member of the study group ( $\mathrm{FE}, \mathrm{MH}, \mathrm{KK}$, or $\mathrm{MMM}$ ) according to the guidelines above. All created examples were then checked by a second researcher ( $\mathrm{FE}, \mathrm{NH}, \mathrm{MH}, \mathrm{KK}$, or $\mathrm{MMM}$ ) to ensure adherence to the guidelines. In instances where the created example did not fulfil the guidelines, the second researcher made edits to ensure that it did. Any edits to the examples were then checked by the researcher who had created the initial example, and if he or she agreed with the new wording, this was accepted as is. If there was disagreement with the new version, then the example was discussed and revised within the group (FE, NH, MH, KK, MMM) until consensus was reached. These consensus-based examples coupled with the self-enactable definitions created in Step 3 made up the draft version of the compendium (v0.3) in supplementary file 7.

\section{Step 5: Qualitative interviews to assess acceptability of techniques}

To examine the prospective acceptability of a subset of 20 techniques among potential end users, qualitative interviews were conducted with individuals recruited via social media. These 20 techniques were selected based on the results of a rating exercise, in which nine experts in self-determination theory rated the likelihood of each technique to impact upon autonomous and controlled forms of motivation ${ }^{43}$. The 20 techniques rated as having the greatest likelihood to increase autonomous forms of motivation and decrease 
controlled forms of motivation were selected for the interviews. Within the interviews, each participant sequentially reviewed a random selection of 12 techniques, including its label, definition and instructive example from v0.3. Following a pilot-tested interview protocol, and after obtaining informed consent, one researcher $(\mathrm{FE})$ asked participants whether the technique definitions and instructive examples were understood as intended, whether participants utilised the techniques themselves, and how they might be able to implement the techniques in their own lives (e.g. to increase physical activity levels). Information on how to improve each technique was also gathered. Interview sessions lasted approximately 75 minutes per participant, and acceptability was assessed using the Theoretical Framework of Acceptability ${ }^{31}$. The University of Helsinki Ethical Review Board in the Humanities and Social and Behavioural Sciences provided a favourable assessment for this work. All portions of this work which involved human participants complied with all relevant ethical regulations. For further description of the methods see supplementary file 11.

\section{Step 6: Review of technique listing and instructive examples by experienced intervention developers}

After compiling the preliminary draft version of the compendium (v0.3), 20 external experts in the development of health behaviour change interventions and/or in the use of taxonomies of behaviour change techniques for coding intervention descriptions were recruited to review it. The expert review aimed to examine: (a) the extent to which each technique was clearly understood from an intervention development standpoint (utility); (b) the extent to which each technique's instructive example was congruent with its definition (congruence); and (c) the extent to which members of the general public would be able to successfully enact each technique based on reading the definition and example (ease of self-enactability). In achieving these aims, we also gathered the experts' qualitative assessments of how each of these aspects could be improved.

A list of 37 potential expert reviewers was identified by the study team, and experts were approached via email to participate. The first 20 who agreed to participate received a link to an online form which allowed them to review of a random selection of 28 to 40 BCTs per participating expert. Each of the BCT definitions and instructive examples to be reviewed was presented on its own page, along with the following three items measuring the (a) utility, (b) congruence, and (c) ease of self-enactability of each technique: (a) "Based on your reading of the definition and example, to what extent do you understand the purpose of this technique and how it could be included as part of a health behaviour change or self-management intervention to influence motivation or change behaviour?", (b) "To what extent does the example reflect the definition?"; and (c) "To what extent would the average layperson be able to successfully use this technique after reading the definition and example?". Experts responded to each item on a 5-point Likert scale with options of 'fully,' 'largely,' 'somewhat,' 'scarcely,' and 'not at all'. If an expert gave a rating of 'somewhat,' 'scarcely,' or 'not at all,' the system prompted him or her to complete follow-up free response items to elicit their opinions on ways in which the utility, congruence, or ease of self-enactability might be improved. Space was also provided for the experts to provide opinions about each technique and the listing as a whole. See supplementary file 8 for verbatim methods of this step.

\section{Step 7: Finalising the classification}

Study team members ( $\mathrm{NH}, \mathrm{MH}, \mathrm{KK}, \mathrm{MMM})$ convened to review all techniques for which the expert review had revealed potential problems with utility, congruence, or ease of self-enactability. All techniques which at least one expert had rated as 'scarcely' or 'not at all,' or which two or more experts had rated as 'somewhat,' in any domain were reviewed. The team reviewed the qualitative responses given during the expert review for each problematic domain of a technique, came to a decision about whether a change to the definition or example was required, and collaboratively brainstormed ways in which utility, congruence, or ease of self-enactability of the technique definition and example could be improved in line with the reviewers' comments. This included re-wording techniques' labels, definitions or examples to improve clarity or precision, defining key terms that are necessary in explaining a definition or example, or adding additional information about the intended or appropriate uses of a technique. In some cases, no action could be taken on the expert's 
536 qualitative responses, as it would have pushed the work beyond its pre-defined boundaries. Changes made

537 during this phase were logged and are presented in supplementary file 10. After these refinements, techniques

538 were re-numbered to group similar techniques and support a logical flow within the listing, resulting in the

539 final compendium (v1.0; supplementary files 1 and 3). Finally, a primer and glossary were written to spell out

540 the purposes of the compendium and to define key terms (supplementary file 2).

\section{Acknowledgments}

543 We would like to thank the expert review participants, whose insights and feedback were vital in

544 carrying out this work.

545

546

\section{Data availability statement}

547 All data generated or analysed during this study are included in this published article (and its

548 supplementary information files).

549

\section{Competing interests statement}

$551 \mathrm{MSH}, \mathrm{WH}$ and MMM are co-authors of existing taxonomies of behaviour change techniques which have informed this work. The authors declare no other competing interests. 
1. Sheeran, P. et al. The impact of changing attitudes, norms, and self-efficacy on health-related intentions and behavior: A meta-analysis. Health Psychol. 35, 1178-1188 (2016).

2. Marcolino, M. S. et al. The Impact of mHealth Interventions: Systematic Review of Systematic Reviews. in JMIR mHealth and uHealth (2018). doi:10.2196/mhealth.8873

3. Ahn, S., Smith, M. L., Altpeter, M., Post, L. \& Ory, M. G. Healthcare Cost Savings Estimator Tool for Chronic Disease Self-Management Program: A New Tool for Program Administrators and Decision Makers. Front. Public Health 3, (2015).

4. Riegel Barbara et al. Self-Care for the Prevention and Management of Cardiovascular Disease and Stroke. J. Am. Heart Assoc. 6, e006997

5. Michie, S. et al. From Theory-Inspired to Theory-Based Interventions: A Protocol for Developing and Testing a Methodology for Linking Behaviour Change Techniques to Theoretical Mechanisms of Action. Ann. Behav. Med. 52, 501-512 (2018).

6. Abraham, C. \& Michie, S. A Taxonomy of Behavior Change Techniques Used in Interventions. Health Psychol. 27, 379-387 (2008).

7. Michie, S., Fixsen, D., Grimshaw, J. M. \& Eccles, M. P. Specifying and reporting complex behaviour change interventions: the need for a scientific method. Implement. Sci. 4, 40 (2009).

8. Michie, S., Johnston, M. \& Carey, R. Behavior Change Techniques. Encycl. Behav. Med. 1-8 (2016). doi:10.1007/978-1-4614-6439-6_1661-2

9. Michie, S. et al. The behavior change technique taxonomy (v1) of 93 hierarchically clustered techniques: Building an international consensus for the reporting of behavior change interventions. Ann. Behav. Med. 46, 81-95 (2013).

10. Kok, G. et al. A taxonomy of behaviour change methods: an Intervention Mapping approach. Health Psychol. Rev. 10, 297-312 (2016).

11. Hardcastle, S. J., Fortier, M., Blake, N. \& Hagger, M. S. Identifying content-based and relational techniques to change behaviour in motivational interviewing. Health Psychol. Rev. 11, 1-16 (2017).

12. William, M. R. \& Rollnick, S. Motivational Interviewing, Third Edition: Helping People Change. (Guilford Press, 2012).

13. Duckworth, A. L., Milkman, K. L. \& Laibson, D. Beyond Willpower: Strategies for Reducing Failures of Self-Control. Psychol. Sci. Public Interest 19, 102-129 (2018). on what people do to manage their motivation and behaviour. (2018). doi:10.31234/osf.io/pa4wg 
590

591

592

593

594

595

596

597

598

599

600

601

602

603

604

605

606

607

608

609

610

611

612

613

614

615

616

617

618

619

620

621

15. Hollands, G. J. et al. The TIPPME intervention typology for changing environments to change behaviour. Nat. Hum. Behav. 1, 0140 (2017).

16. Hartmann-Boyce, J., Aveyard, P., Koshiaris, C. \& Jebb, S. A. Development of tools to study personal weight control strategies: OxFAB taxonomy. Obesity 24, 314-320 (2016).

17. Richardson, M. J., Dale, R. \& Marsh, K. L. Complex dynamical systems in social and personality psychology: Theory, modeling, and analysis. in Handbook of research methods in social and personality psychology, 2nd ed 253-282 (Cambridge University Press, 2014).

18. Shiell, A., Hawe, P. \& Gold, L. Complex interventions or complex systems? Implications for health economic evaluation. BMJ 336, 1281-1283 (2008).

19. Bellg, A. J. et al. Enhancing treatment fidelity in health behavior change studies: best practices and recommendations from the NIH Behavior Change Consortium. Health Psychol. Off. J. Div. Health Psychol. Am. Psychol. Assoc. 23, 443-451 (2004).

20. Knittle, K., De Gucht, V., Hurkmans, E., Vlieland, T. V. \& Maes, S. Explaining Physical Activity Maintenance after a Theory-Based Intervention among Patients with Rheumatoid Arthritis: Process Evaluation of a Randomized Controlled Trial. Arthritis Care Res. 68, (2016).

21. Hankonen, N. et al. Which Behavior Change Techniques are Associated with Changes in Physical Activity, Diet and Body Mass Index in People with Recently Diagnosed Diabetes? Ann. Behav. Med. 49, 7-17 (2015).

22. Burke, L. E., Wang, J. \& Sevick, M. A. Self-Monitoring in Weight Loss: A Systematic Review of the Literature. J. Am. Diet. Assoc. 111, 92-102 (2011).

23. Tims, M., Bakker, A. B. \& Derks, D. The impact of job crafting on job demands, job resources, and well-being. J. Occup. Health Psychol. 18, 230-240 (2013).

24. Brick, N., Maclntyre, T. \& Campbell, M. Attentional focus in endurance activity: new paradigms and future directions. Int. Rev. Sport Exerc. Psychol. 7, 106-134 (2014).

25. Van Raalte, J. L., Vincent, A. \& Brewer, B. W. Self-talk: Review and sport-specific model. Psychol. Sport Exerc. 22, 139-148 (2016).

26. Maes, S. \& Karoly, P. Self-regulation assessment and intervention in physical health and illness: A review. Appl. Psychol. 54, 267-299 (2005).

27. Teixeira, P. J. et al. Classification of Techniques Used in Self-Determination Theory-Based Interventions in Health Contexts: An Expert Consensus Study. (2018).

28. Fleiss, J. L. Measuring nominal scale agreement among many raters. Psychol. Bull. 76, 378-382 (1971). 
29. Black, N. et al. Enhancing Behavior Change Technique Coding Methods: Identifying Behavioral Targets and Delivery Styles in Smoking Cessation Trials. Ann. Behav. Med. doi:10.1093/abm/kay068

30. Lorencatto, F., West, R., Bruguera, C., Brose, L. S. \& Michie, S. Assessing the Quality of Goal Setting in Behavioural Support for Smoking Cessation and its Association with Outcomes. Ann. Behav. Med. 50, 310-318 (2016).

31. Sekhon, M., Cartwright, M. \& Francis, J. J. Acceptability of healthcare interventions: an overview of reviews and development of a theoretical framework. BMC Health Serv. Res. 17, 88 (2017).

32. Urrea, B. et al. Mobile Health Initiatives to Improve Outcomes in Primary Prevention of Cardiovascular Disease. Curr. Treat. Options Cardiovasc. Med. 17, 59 (2015).

33. Schroeder, J. \& Fishbach, A. How to motivate yourself and others? Intended and unintended consequences. Res. Organ. Behav. 35, 123-141 (2015).

34. Guerdjikova, A. I., Mori, N., Casuto, L. S. \& McElroy, S. L. Binge Eating Disorder. Psychiatr. Clin. 40, 255-266 (2017).

35. Michie, S. et al. A refined taxonomy of behaviour change techniques to help people change their physical activity and healthy eating behaviours: the CALO-RE taxonomy. Psychol. Health 37-41 (2011). doi:10.1080/08870446.2010.540664

36. Marques, M. \& Hagger, M. S. Identifying the Active Components of Self-Determination TheoryBased Health Behavior Change Interventions: An Expert Consensus Study. (2018). doi:None

37. Brabham, D. C. Crowdsourcing as a Model for Problem Solving: An Introduction and Cases. Convergence 14, 75-90 (2008).

38. Carey, R. N. et al. Behavior Change Techniques and Their Mechanisms of Action: A Synthesis of Links Described in Published Intervention Literature. Ann. Behav. Med. (2018).

39. Johnston, M. et al. Linking behavior change techniques and mechanisms of action: Triangulation of findings from literature synthesis and expert consensus. (2018). doi:10.31234/osf.io/ur6kz

40. Greaves, C. J. Uptake of Behavior Change Techniques - a Key Focus for Process Analysis and for Intervention Delivery: a Comment on Hankonen et al. Ann. Behav. Med. 49, 1-2 (2015).

41. Bradbury, K., Watts, S., Arden-Close, E., Yardley, L. \& Lewith, G. Developing Digital Interventions: A Methodological Guide. Evidence-Based Complementary and Alternative Medicine (2014). doi:10.1155/2014/561320

42. de Vries, H. \& Brug, J. Computer-tailored interventions motivating people to adopt health promoting behaviours: introduction to a new approach. Patient Educ. Couns. 36, 99-105 (1999).

43. Deci, E. L. \& Ryan, R. M. The 'what' and 'why' of goal pursuits: Human needs and the selfdetermination of behavior. Psychol. Inq. 11, 227-268 (2000). 